Article

\title{
Assigning the Origin of Microbial Natural Products by Chemical Space Map and Machine Learning
}

\author{
Alice Capecchi and Jean-Louis Reymond * (D) \\ Department of Chemistry and Biochemistry, University of Bern, Freiestrasse 3, 3012 Bern, Switzerland; \\ alice.capecchi@dcb.unibe.ch \\ * Correspondence: jean-louis.reymond@dcb.unibe.ch
}

Received: 2 September 2020; Accepted: 25 September 2020; Published: 28 September 2020

\begin{abstract}
Microbial natural products (NPs) are an important source of drugs, however, their structural diversity remains poorly understood. Here we used our recently reported MinHashed Atom Pair fingerprint with diameter of four bonds (MAP4), a fingerprint suitable for molecules across very different sizes, to analyze the Natural Products Atlas (NPAtlas), a database of 25,523 NPs of bacterial or fungal origin. To visualize NPAtlas by MAP4 similarity, we used the dimensionality reduction method tree map (TMAP). The resulting interactive map organizes molecules by physico-chemical properties and compound families such as peptides and glycosides. Remarkably, the map separates bacterial and fungal NPs from one another, revealing that these two compound families are intrinsically different despite their related biosynthetic pathways. We used these differences to train a machine learning model capable of distinguishing between NPs of bacterial or fungal origin.
\end{abstract}

Keywords: natural products; databases; cheminformatics; chemical space; visualization; molecular fingerprints; machine learning; support vector machine; origin classification

\section{Introduction}

Natural products (NPs) of microbial origin are an important source of drugs. Numerous examples of antibiotic, antifungal, immunosuppressive, anti-inflammatory, and anti-cancer agents on the market originate from fungi or bacteria [1]. A notable effort has been made to explore the known and virtual chemical space of microbial NPs and NPs in general [2-5]. Furthermore, machine learning (ML) has been extensively applied to natural product structures, for example, to classify limonoids and protolimonoids [6], to establish the structural class of a natural product with its NMR data [7], to learn estimates of natural product conformational energies [8], to generate derivates of NPs or compounds with natural product characteristics [9-11], to predict meridian in Chinese traditional medicine [12], and to elucidate the biological effects of natural products [13]. The recently published Natural Products Atlas (NPAtlas) is a collection of 25,523 NPs of fungal and bacterial origin [14]. Among other tools, the NPAtlas website (https://www.npatlas.org/joomla/) provides a global view of the database in a spherical representation. To generate this view, the NPAtlas entries are clustered by Dice similarity [15] using the substructure fingerprint ECFP4 (an extended connectivity fingerprint with a diameter of four bonds) [16]. The resulting clusters are grouped in nodes, which are arranged in a spherical plot where the position of each node is determined by molecular formulas. While this representation provides interesting insights into the composition of the NPAtlas, individual compounds cannot be visualized in the global overview but only within clusters, therefore, comparing compounds across two different clusters is not possible.

A defining feature of NPAtlas is that NPs featured in this database span across a broad range of sizes, with the largest NPs reaching up to almost $3 \mathrm{kDa}$ (Figure S1). We showed recently that the ECFP4 fingerprint, although well suited for small molecule drugs, performed poorly with larger molecules 
typically found in NP collections such as lipids, oligosaccharides, and peptides [17]. To address this limitation, we recently investigated molecular fingerprints combining the concept of atom pairs [18], which is well suited to analyze large molecules such as proteins and peptides [19-22], with extended connectivity substructures and bit compression using MinHash as used in the substructure fingerprint MHFP6 [23], and proposed the MinHashed atom pair fingerprint with a diameter of four bonds (MAP4) as an optimal molecular fingerprint to analyze molecules of very different sizes [17].

Here we asked the question of whether analyzing NPAtlas using MAP4 might provide new insights into the composition of this collection. To organize molecules according to their MAP4 similarity, we used TMAP, a recently reported dimensionality reduction method suitable to analyze very large high-dimensional datasets [24]. TMAP performs better for the visualization of large high-dimensional data sets than other dimensionality reduction methods such as t-SNE [25] or UMAP [26]. Furthermore, TMAP is particularly well suited to analyze databases of molecules associated with MinHashed fingerprints.

\section{Materials and Methods}

\subsection{NPAtlas Dataset}

The December 2019 version of the NPAtlas was used. This version of the database contains 25,523 entries, 15,759 of fungal origin, and 9764 entries of bacterial origin, with no entry sharing bacterial and fungal origin. For each compound, a simplified molecular-input line-entry system (SMILES), molecular weight (MW), origin (fungal or bacterial), and the DOI of the associated publication were downloaded. For the MAP4 fingerprint calculation, the SMILES were canonicalized [27] and the stereochemistry was removed using the RDKit toolkit [28]. After removing stereochemistry, the NPAtlas counts 23,928 unique SMILES and 76 entries common among both origins.

\subsection{MAP4 Fingerprint}

The MAP4 fingerprint combines the circular substructure and atom pair fingerprints concepts. MAP4 encodes each atom pair in a molecule as the SMILES of the circular substructure of radii 1 and 2 around both atoms and the distance in bonds that separates them. The resulting set of strings is hashed to integers using the SHA-1 algorithm [29] and MinHash scheme [30]. The obtained MAP4 fingerprint is an array of unsorted numbers, where each feature is characterized by its value and its position in the array (index). MAP4 perceives substructure details while maintaining a global overview; therefore, it is suitable to describe molecular structures across different sizes. The similarity between two MAP4 fingerprints a and b was calculated: (1) counting of elements with the same value and the same index across $a$ and $b$, and (2) dividing the obtained value by the number of elements of fingerprint a. The similarity between two MinHashed MAP4 fingerprints calculated as described above is an estimation of the Jaccard Similarity between the two non-MinHashed objects [30]. For a detailed explanation if the MAP4 implementation and benchmark, please refer to our recent publication [17]. The 1024-dimensions MAP4 fingerprint of all NPAtlas entries was calculated using canonical SMILES without stereochemistry information.

\subsection{TMAP Layout}

The TMAP layout was calculated from the MAP4 fingerprint dataset using the open-source implementation of TMAP [24]. In short, the indices generated by the MinHash procedure of the MAP4 calculation were used to create a locality-sensitive hashing (LSH) forest [31] of $n$ trees. For each NPAtlas entry, the $k$ approximate nearest neighbors (NNs) in the MAP4 feature space are then extracted from the LSH forest to form a graph in which nodes are the structures and edges are the NN relationships weighted by the fingerprint distance. The Kruskal's algorithm was then applied to remove cycles and to find the path with the lowest total distance between all molecules in the graph [32]. Finally, 
Fearun [33] was used to interactively display the obtained minimum spanning tree. In this study, we set $\mathrm{n}=32$ and $k=20$.

\subsection{Properties Calculation}

For all NPAtlas entries, the number of hydrogen bond acceptors (HBA) and hydrogen bond donors (HBD), $\log$ P following Crippens approach (AlogP) [34], topological polar surface area (TPSA), and fraction of sp3 carbon (fsp3C) were calculated with RDKit. The boiling point was calculated using

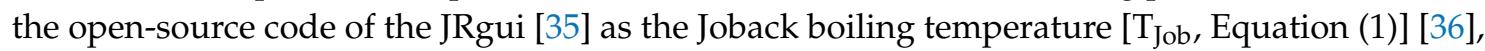

$$
T_{J o b}=198.2+\sum_{i} N_{i} t_{b i}
$$

where $N_{i}$ is the occurrence of a functional group in the molecule, and $t_{b i}$ is its empirically obtained contribution value. Molecules that violated more than one Lipinski rules [37] were labeled as non-Lipinski. To identify glycosylated and/or peptidic structures, Daylight [38] SMARTS language was used. SMILES arbitrary target specification (SMARTS) were used with RDKit to identify NPAtlas entries containing a dipeptide substructure, defined as "[NX3,NX4+][CH1,CH2][CX3] $=[\mathrm{OX} 1])[\mathrm{NX} 3, \mathrm{NX} 4+][\mathrm{CH} 1, \mathrm{CH} 2][\mathrm{CX} 3](=[\mathrm{OX} 1])[\mathrm{O}, \mathrm{N}]$ ”, or a glycoside substructure, defined as "[CR][OR][CHR]([OR0,NR0])[CR]".

\subsection{TMAP Color Gradients}

The calculated properties were used to color the generated TMAP. For a clearer color gradient, some of the highest and lowest displayed values of the non-ranked properties have been adjusted. All MW values $\geq 1000 \mathrm{Da}$ are displayed as $1,000 \mathrm{Da}$, all boiling point values $\geq 2000 \mathrm{~K}$ are displayed as $2000 \mathrm{~K}$, all HBD count values $\geq 10$ are displayed as 10 , all AlogP values $\geq 8$ are displayed as 8 , all AlogP values $\leq-2$ are displayed as -2 , and all TPSA values $\geq 500$ are displayed as 500 . The color-codes of the ranked property values were obtained by average ranking them using SciPy [39]. In average ranking, if two or more values have the same rank, the average rank of the tied values is assigned to each of them. For details on TMAP please refer to the related publication [24].

\subsection{Support Vector Machine (SVM) and k-Nearest Neighbor ( $k$-NN) Classifiers}

The $k$-nearest neighbor $(k-\mathrm{NN})$ algorithm is a simple ML method that predicts the query to belong to the class most found amongst its $k$ nearest neighbors. A support vector machine (SVM) represents a more complex ML approach; an SVM maps its input into a high-dimensional feature space and tries to find the best separation between two classes, such as they entirely lay on the opposite side a hyperplane. To do so, the SVM maximizes the margin between the closest points, known as support vectors, and the hyperplane. Mapping features explicitly into a higher dimensional space is computationally expensive and not feasible even for small datasets. To avoid it the SVM uses the so-called "kernel trick", which essentially uses a similarity matrix of the input data instead of the input itself; this allows the SVM to define the hyperplane and the support vectors in a less expensive manner [40]. In cheminformatics, both $k$-NN and SVM inputs can range from SMILES to various molecular descriptors. For this work, three classifiers were implemented: a MAP4 based $k$-NN (MAP4 $k$-NN), a MAP4 based SVM (MAP4 SVM), and an SVM based on physico-chemical properties (physchem SVM).

The MAP4 SVM and MAP4 $k$-NN classifiers were implemented as follows. The canonicalized SMILES without stereochemistry information used to generate the TMAP were made unique, and they were assigned to training or test set with a 50\% random split. The 35 unique SMILES of the 76 entries common between both origins were randomly assigned to one origin. Both classifiers were trained using MAP4 fingerprints. In both cases, the class weights were inversely proportional to the class frequency, and their hyperparameter was optimized using a 5-fold cross-validation. During the 5-fold cross-validation, $20 \%$ of the training set was left out as a validation set, and the final set of parameters 
maximized the ROC AUC on the validation set. For the SVM classifier, the hyperparameter C was optimized among the values $0.1,1,10,100$, and 1000, resulting in $C=10$. The SVM utilized a custom kernel that calculated the similarity matrix between two MAP4 fingerprints. Platt scaling [41] was used to obtain probabilistic prediction values. For the $k$-NN model, the number of nearest neighbors $k$ was optimized among the values 5, 7, 9, and 11, resulting in $k=7$. As a distance metric between two MAP4 fingerprints, the $k$-NN classifier used one minus the similarity between the two fingerprints.

The physchem SVM model was trained with the same training/test split, but using the MW, fsp3C, HBA, HBD, AlogP, TPSA, and calculated boiling point as input. The properties were scaled to zero mean and unit variance. A radial basis function (RBF) kernel [42] was used, and the hyperparameters $C$ and $\gamma$ were optimized with a grid search. $C$ was optimized considering the values $0.1,1,10,100$, and 1000, resulting in $C=10$, and $\gamma$ was optimized considering the values $0.01,0.1,1,10$, and 100 , resulting in $\gamma=1$.

For the evaluation of the classifiers, we considered the class "bacterium" to be the positive class and the class "fungus" to be the negative one. All SVM and the $k$-NN classifiers were implemented using scikit-learn [43], and all not mentioned hyperparameters were used in their default values. The source code for all classifiers can be found at https://github.com/reymond-group/MAP4-ChemicalSpace-of-NPAtlas.

\subsection{Classifiers Evaluation Metrics}

ROC AUC is the area under the ROC curve, and the ROC curve is obtained by plotting the true positive rate (TPR) against the false positive rate (FPR):

$$
\begin{aligned}
& T P R=\frac{T P}{T P+F P} \\
& F P R=\frac{F P}{T P+F P}
\end{aligned}
$$

where $T P$ stands for true positives, $T N$ for true negatives, $F P$ for false positives, and $F N$ for false negatives predicted by the classifier.

The $F 1$ score is defined as the harmonic mean of precision and recall:

$$
\begin{gathered}
\text { Precision }=T P R \\
\text { Recall }=\frac{T P}{T P+F N} \\
F 1 \text { score }=2 \times \frac{(\text { Precision } \times \text { Recall })}{(\text { Precision }+ \text { Recall })}
\end{gathered}
$$

The balanced accuracy is defined as:

$$
\text { Balanced accuracy }=\frac{T P R+\frac{T N}{T N+F N}}{2}
$$

The Matthews correlation coefficient (MCC) is a correlation between the observed and the predicted class and it is defined as:

$$
M C C=\frac{T P \times T N-F P \times F N}{\sqrt{(T P+F P)(T P+F N)(T N+F P)(T N+F N)}}
$$

In all metrics, the probabilistic prediction values were converted into binary classification values using a threshold of 0.5 . 


\section{Results and Discussion}

\subsection{The TMAP of NPAtlas}

The 25,523 structures in NPAtlas were downloaded and encoded using the MAP4 fingerprint, which is well suited to analyze molecules across different sizes such as those in NPAtlas ranging between 70 and 2900 Da in MW (Table 1, Figure S1, method Sections 2.1 and 2.2). The generated dataset was then visualized using TMAP, which represents the minimum spanning tree connecting nearest neighbors, here according to the MAP4 similarity measured as Jaccard distance (Figure S2, see method Section 2.3 for details). To understand how the NPs in NPAtlas are organized on the MAP4 TMAP, we generated color codes based on various physico-chemical descriptors, as well as on categorical classification by compound type and observed or predicted origin (Table 1, method Sections 2.4 and 2.5, Figures S3-S5, https://tm.gdb.tools/map4/).

Table 1. Calculated properties of NPAtlas molecules available as TMAP color-codes.

\begin{tabular}{|c|c|c|c|c|c|}
\hline Property & Min. Value & Max. Value & $25 \%$ Quantile & $50 \%$ Quantile & 75\% Quantile \\
\hline Molecular weight ${ }^{A}$ & 70.1 & $2901.3\left(1000^{\mathrm{F}}\right)$ & 292 & 408.9 & 562.6 \\
\hline Sp3 C fraction $\mathrm{A}$ & 0.0 & 1.0 & 0.4 & 0.6 & 0.7 \\
\hline HBD count ${ }^{A, C}$ & 0 & $47\left(10^{\mathrm{F}}\right)$ & 3 & 2 & 4 \\
\hline $\mathrm{A} \log \mathrm{P} A, \mathrm{D}$ & $-28.9\left(-2^{G}\right)$ & $33.8\left(8^{\mathrm{F}}\right)$ & 1.2 & 2.5 & 4.1 \\
\hline TPSA A,E & 0.0 & $1135.81\left(500^{\mathrm{F}}\right)$ & 69.64 & 99.66 & 152.8 \\
\hline Is Lipinski & \multicolumn{5}{|c|}{ Categorical: yes/no } \\
\hline Substructures ${ }^{\mathrm{I}}$ & \multicolumn{5}{|c|}{$\begin{array}{c}\text { Categorical: contains dipeptide moiety/contains glycoside moiety/contains dipeptide and } \\
\text { glycoside moieties }\end{array}$} \\
\hline Origin & \multicolumn{5}{|c|}{ Categorical: Bacterial/Fungal } \\
\hline MAP4 SVM ${ }^{\mathrm{J}}$ prediction & \multicolumn{5}{|c|}{ Categorical: Bacterial/Fungal } \\
\hline MAP4 SVM J performances & \multicolumn{5}{|c|}{ Categorical: correct/wrong } \\
\hline
\end{tabular}

Inspecting the colored TMAPs reveals that molecules are organized by structural features. For example, inspecting the TMAP colored by MW shows that most of the high MW compounds (MW $\geq 1000 \mathrm{Da}, 6.8 \%$ of NPAtlas) belong to three structural families, namely peptides type compounds (minimal substructure: dipeptide), glycosides (minimal substructure: cyclic N- or O-acetal) and glycopeptides (both substructures present) (Table 2, Figure 1A and Figure S1). Typical examples of such large NPs are shown in Figure 2, featuring the cyclic peptides jizanpeptin A (NPA022688, bacterial) [44] and arbumelin (NPA020152, fungal) [45], the glycosides butirosin A (NPA009292, bacterial) [46] and quinofuracin A (NPA005440, fungal) [47], and the glycopeptides cycloaspeptide F (NPA000712, the only fungal glycopeptide in NPAtlas) [48] and orienticin D (NPA021348, bacterial) [49].

Table 2. NPAtlas entries and unique publications number according to the origin and molecular weight.

\begin{tabular}{|c|c|c|}
\hline & Fungal $^{A}$ & Bacterial $^{\mathrm{A}}$ \\
\hline NPAtlas entries ( $\geq 1000 \mathrm{Da})$ & $15,759(347)$ & 9764 (1392) \\
\hline Unique publications ${ }^{\mathrm{B}}$ & $6110(145)$ & $4653(711)$ \\
\hline Peptides $(\geq 1000 \mathrm{Da})^{\mathrm{C}}$ & $722(311)$ & $2144(901)$ \\
\hline Glycosides $(\geq 1000 \mathrm{Da})^{\mathrm{D}}$ & $814(12)$ & $1616(421)$ \\
\hline Glycopeptides $(\geq 1000 \mathrm{Da})^{\mathrm{E}}$ & $1(0)$ & $112(89)$ \\
\hline Aromatic NPs $(\geq 1000 \mathrm{Da})^{\mathrm{F}}$ & $1322(0)$ & $800(31)$ \\
\hline Aliphatic NPs ( $\geq 1000 \mathrm{Da})^{\mathrm{G}}$ & $2184(59)$ & $1366(220)$ \\
\hline
\end{tabular}

A Natural product origin. ${ }^{B}$ Number of unique publications used for the extraction of all NPAtlas entries ${ }^{C}$ Containing a dipeptide moiety. ${ }^{\mathrm{D}}$ Containing a glycoside moiety. ${ }^{\mathrm{E}}$ both glycoside and dipeptide moiety. ${ }^{\mathrm{F}}$ fsp3C $<$ 0.2. $\mathrm{G}$ fsp $3 \mathrm{C}>0.8$. 


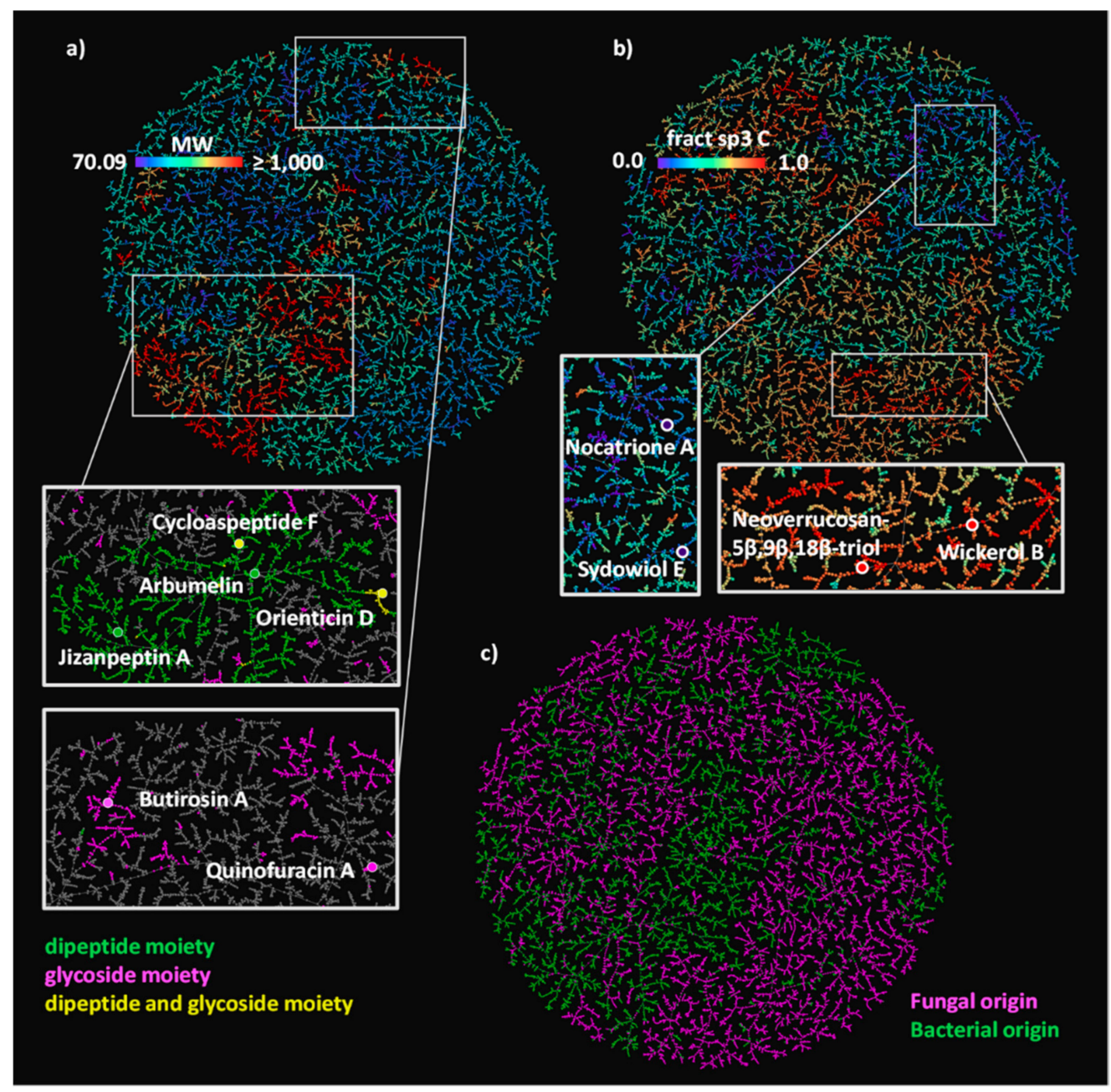

Figure 1. (A) NPAtlas MAP4 TMAP colored by MW, with a rainbow scale where the lowest values are purple, and the highest values are red. Two areas of the map are zoomed and colored by SMARTS substructure match: compounds containing a dipeptide moiety are highlighted in green, compounds containing a glycoside moiety are highlighted in magenta, compounds containing both moieties are highlighted in yellow; six examples of NPAtlas entries are reported with the same color code. (B) The NPAtlas MAP4 TMAP colored by fsp3C with a rainbow scale where the lowest values are purple, and the highest values are red. A low and a high fsp3C area of the map are zoomed, and two examples of polyphenols and of terpenoids are reported. (C) The NPAtlas MAP4 TMAP colored by a microbial origin classification, the compounds originated from fungi are colored in magenta, the compounds produced by bacteria are colored in green.

Another striking insight is provided by inspecting the TMAP colored by the fraction of sp3 carbons (fsp3C, Figure 1B), which allows the identification of areas rich in aromatic polyphenols with very low fsp3C, such as nocatrione A (NPA014210, bacterial) [50] and sydowiol E (NPA001030, fungal) [51], as well as areas populated by terpenoids with very high fsp3C such as neoverrucosane diterpenoids (e.g., neoverrucosan-5 $\beta, 9 \beta, 18 \beta$-triol, NPA001820, bacterial) [52] and the anti-influenza virus diterpene wickerol B (NPA008911, fungal) [53]. The structures of these compounds are shown in Figure 2.

The TMAP not only organizes molecules by structural features, but also separates molecules according to their origin, with fungal and bacterial NPs forming well-defined groups across the TMAP (Figure 1C). This separation is striking because biosynthetic pathways in bacteria and fungi are generally similar, and because the different compound families contain NPs of both bacterial and fungal origin (Table 2). 

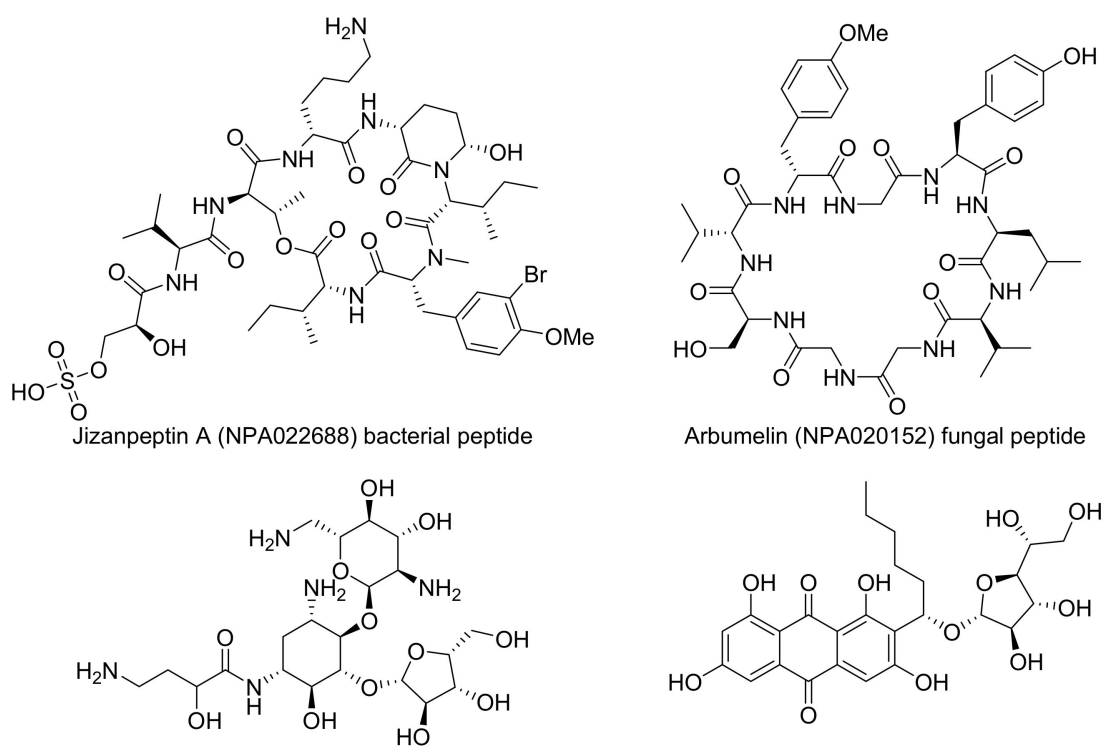

Butirosin A (NPA009292) bacterial glycoside
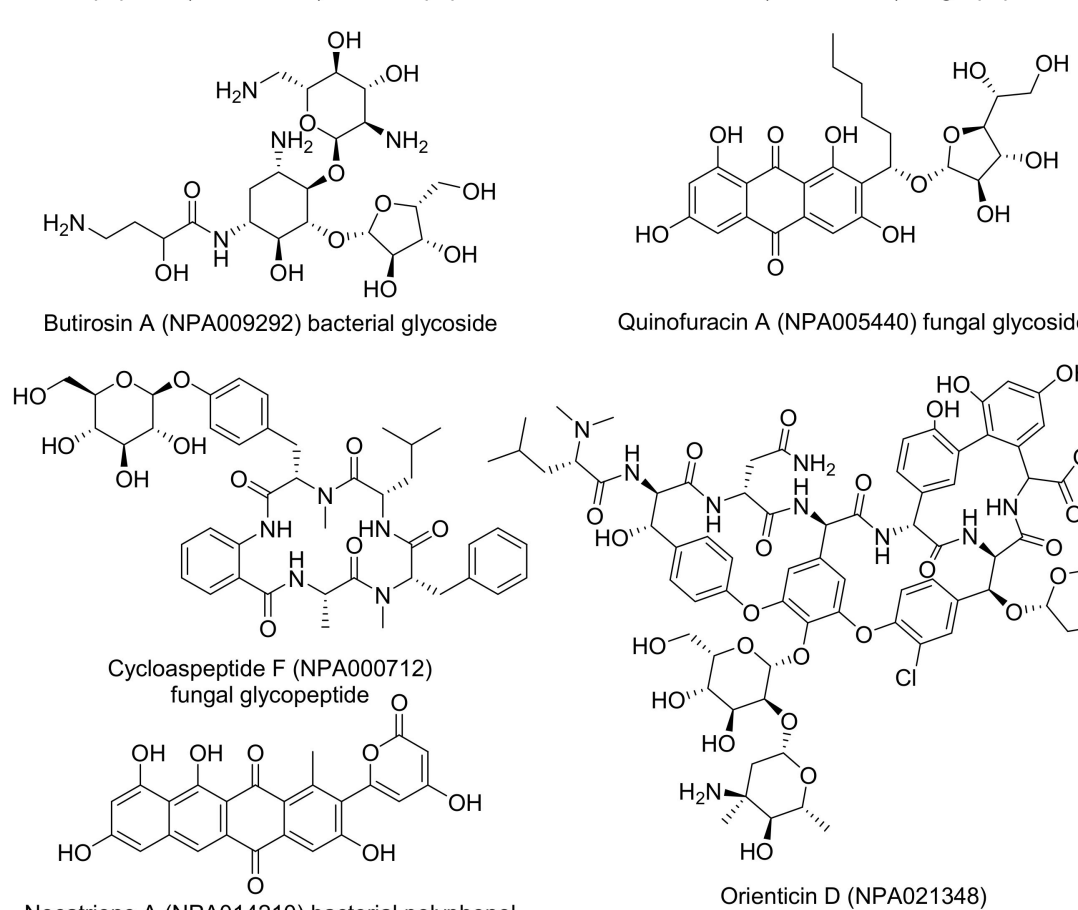

Quinofuracin A (NPA005440) fungal glycoside

Nocatrione A (NPA014210) bacterial polyphenol<smiles>Cc1cc(O)c(Oc2c(O)cc(C)cc2Oc2c(O)cc(C)c(O)c2Oc2c(O)cc(C)cc2O)c(O)c1</smiles>
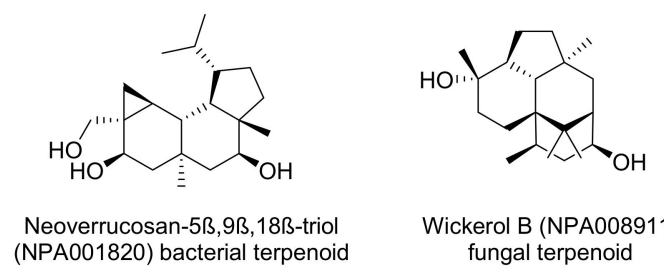

Wickerol B (NPA008911) fungal terpenoid

Figure 2. The structural formula of natural product examples selected from the TMAPs in Figure 1.

\subsection{Distinguishing Between Bacterial and Fungal NPs}

The separation between bacterial and fungal NPs on the MAP4 TMAP and the fact that the map also separates NPs by physico-chemical descriptor values suggested to us that ML models trained either with the MAP4 fingerprint or simply with physico-chemical descriptors might be able to distinguish between NPs of bacterial or fungal origin. We investigated SVM and $k-\mathrm{NN}$ models since this type of ML models are generally well suited for classifying bioactive molecules [54]. We considered both an SVM and a $k$-NN model with MAP4, and only an SVM model with physico-chemical descriptors, and we evaluated their performance on the test set (see method Section 2.6).

The MAP4 SVM was the best performing model with an area under the receiver operating characteristic curve (ROC AUC) of 0.97 , an F1 score of 0.91 , a balanced accuracy of 0.93 , and a Matthews correlation coefficient (MCC) of 0.86 (Table 3). The MAP4 $k$-NN classifier also had excellent evaluation metrics with an accuracy of 0.90 and an MCC of 0.8 , suggesting the high performance of the MAP4 
SVM classifier might depend on a nearest neighbor effect. On the other hand, the physchem SVM performed significantly worse than the MAP4 based classifiers and was only partially capable of distinguishing between bacterial and fungal NPs (F1 score and a balanced accuracy above 0.7). This suggests that successful classification requires a model distinguishing between specific substructures and not only overall molecular properties. For closer inspection, the prediction (fungal or bacterial origin) and the performance (correct or wrong) of the best performing classifier (MAP4 SVM) are color-coded on the MAP4 TMAP of NPAtlas (Figure S5).

Table 3. SVM and $k$-NN classifier's performance on the test set.

\begin{tabular}{ccccc}
\hline Classifier & ROC AUC $^{\mathbf{A}}$ & F1 Score $^{\mathbf{A}}$ & Balanced Accuracy $^{\text {A }}$ & MCC $^{\mathbf{A}}$ \\
\hline MAP4 SVM $^{\text {B }}$ & 0.97 & 0.91 & 0.93 & 0.86 \\
MAP4 $k$-NN $^{\text {C }}$ & 0.96 & 0.88 & 0.90 & 0.81 \\
Physchem SVM $^{\text {D }}$ & 0.86 & 0.73 & 0.78 & 0.56 \\
\hline
\end{tabular}

${ }^{\text {A }}$ Area under the receiver operating characteristic curve (ROC AUC), F1 score, balanced accuracy, and MCC are metrices used to evaluate a machine learning model. MCC can assume values from -1 to 1 , all other parameters can assume values from 0 to 1 , and in all cases 1 is a perfect classification. Refer to Section 2 for details. ${ }^{B}$ SVM classifier trained with the MAP4 fingerprint. ${ }^{C} k$-NN classifier trained with the MAP4 fingerprint. ${ }^{D}$ SVM trained with physiochemical properties.

\subsection{Predicting the Origin of Newly Discovered NPs}

Discussion with natural product chemists informed us that assigning NPs to their origin only from its chemical structure is not trivial, and can be problematic when isolating a new NP due to the occurrence of endosymbiosis, i.e., the fact that bacteria often live as symbionts within larger organisms [51,55]. We therefore asked the question whether our MAP4 SVM classifier would correctly predict the origin of NPs newly reported in 2020 and which are not part of NPAtlas (Table 4). To our delight, the classifier correctly predicted the fungal origin for the newly reported epicospirocins 1 [56], penicimeroterpenoid A [57], and rhizolutin [58], as well as the bacterial origin of the recently reported bosamycin A [59]. The correct origin assignment is probably related to the presence of structurally similar NPs within the NPAtlas training set, illustrated here by the MAP4 nearest-neighbor NPs aspermicrone A [60], isocitreohybridone H [61], Monacolin K [62], and AIP I [63] (Figure 3).

Table 4. MAP4 SVM classification of new microbial natural products and of Phakefustatin A.

\begin{tabular}{cccc}
\hline Natural Product & $\begin{array}{c}\text { MAP4 SVM A } \\
\text { Fungal, Bacterial }\end{array}$ & $\begin{array}{c}\text { Training Set } \\
\text { Nearest Neighbor (NN) }\end{array}$ & JD from NN $^{\text {B }}$ \\
\hline Epicospirocin 1 & $0.99,0.01$ & Aspermicrone A (NPA024935) & 0.66 \\
Penicimeroterpenoid A & $1.0,0.0$ & Isocitreohybridone H (NPA016454) & 0.63 \\
Rhizolutin & $0.83,0.17$ & Monacolin K (NPA009354) & 0.80 \\
Bosamycin A & $0.04,0.96$ & AIP I (NPA010987) & 0.77 \\
Phakefustatin A & $0.12,0.88$ & Samoamide A (NPA022212) & 0.68 \\
\hline
\end{tabular}

A Predicted origin: fungal or bacterial. ${ }^{B}$ Approximated Jaccard distance (JD), see Section 2 for details from the training set NN.

When challenged with the recently reported NP phakefustatin A, isolated from the marine sponge Phakellia fusca [64] (Figure 3), the MAP4 SVM classifier predicted a bacterial origin (Table 4). Indeed, the NPAtlas training set contained closely related NPs of bacterial origin, such as the MAP4 NN Samoamide A [65] (Figure 3). Although phakefustatin A was isolated from a marine sponge, our prediction is probably correct because many marine sponges contain endosymbiotic bacteria, which can make up to $60 \%$ of the sponge biomass and are often responsible for the production of metabolites [66]. More specifically, it is known that Phakellia fusca coexists with diverse actinobacteria which have been held responsible for the production of many bioactive NPs found in the sponge [67].

While the example above might be a case of endosymbiosis and potential origin misclassification, it must be noted that our MAP4 SVM classifier can only label NPs as of bacterial or fungal origin. 
In fact, our classifier mistakenly assigns such classification to well-known non-microbial NPs (Table S1, Figure S6). An extension of our analysis to non-microbial natural products could be of interest, however, the task cannot be completed due to a lack of annotated public datasets for NPs of diverse origins [68,69].

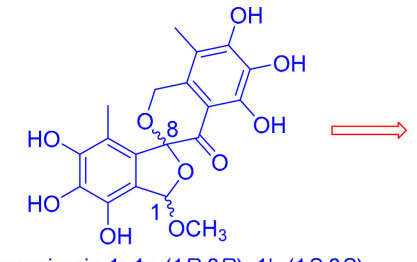

epicospirocin 1: 1a $(1 R, 8 R), 1 \mathrm{~b}(1 S, 8 S)$ (prediction: fungal)

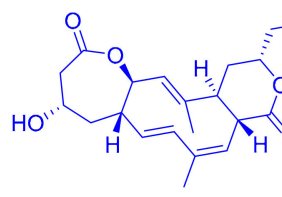
rhizolutin
(prediction: fungal)<smiles>C=CO</smiles>
(NN of epicospirocin 1, fungal)

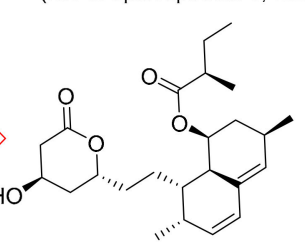

monacolin K (NPA009354) (NN of rhizolutin, fungal) aspermicrone A (NPA024935)

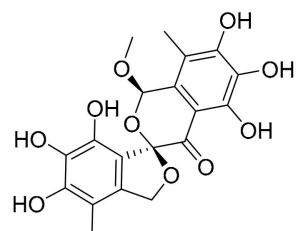

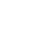

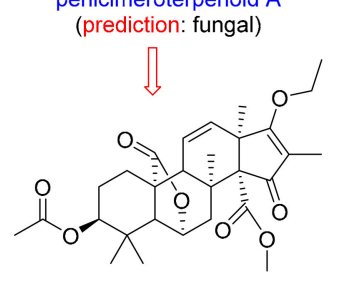

isocitreohybridone $\mathrm{H}$ (NPA016454) (NN of penicimeroterpenoid $A$, fungal)

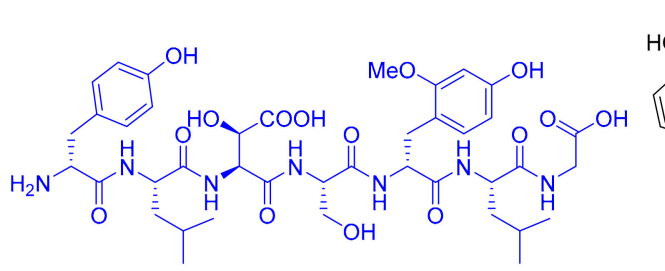
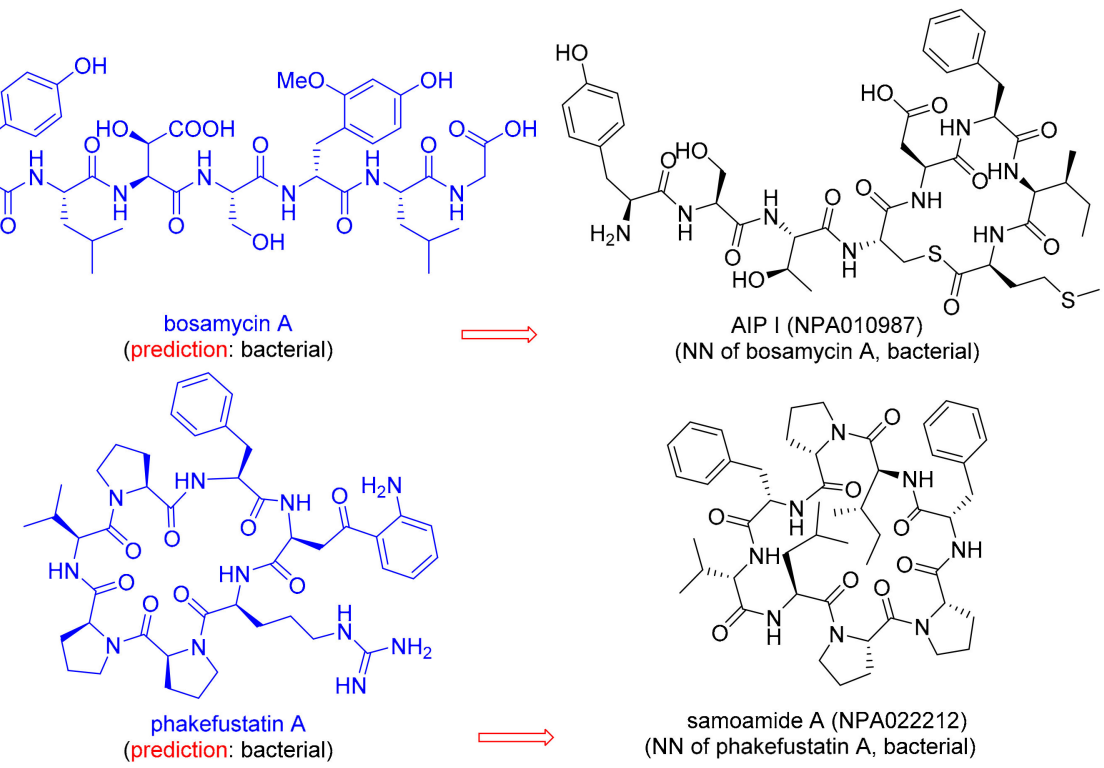

(NN of bosamycin $A$,

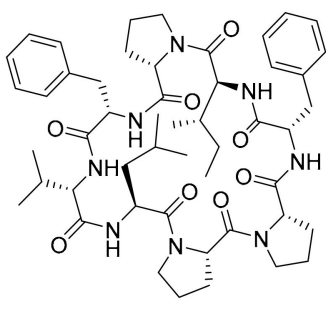

samoamide A (NPA022212) (NN of phakefustatin $A$, bacterial)

Figure 3. Examples of natural products reported in 2020, absent from NPAtlas, annotated with their predicted origin, and connected to its MAP4 NN in the training set.

\section{Conclusions}

In summary, we showed that mapping the 25,523 NPs reported in NPAtlas as a MAP4 TMAP organizes molecules by physico-chemical properties and by substructures and thereby provides an unprecedented insight into the composition of this collection. Most strikingly, the map separates the different NPs according to their bacterial or fungal origin. Furthermore, a SVM model trained with the MAP4 fingerprint dataset performs remarkably well in distinguishing between fungal and bacterial NPs. The classifier can be of aid where the origin of a natural product is unknown, especially when the molecule is isolated from a symbiotic complex. The MAP4 TMAP of NPAtlas is accessible at https://tm.gdb.tools/map4/, and the source code is available at https://github.com/reymond-group/ MAP4-Chemical-Space-of-NPAtlas.

Supplementary Materials: The following are available online at http://www.mdpi.com/2218-273X/10/10/1385/s1, Figure S1: Physico-chemical properties distribution across the NPAtlas entries. Figure S2: Approximated Jaccard 
distance from the top 20 NNs of all NPAtlas entries. Figure S3: MAP4 TMAP of NPAtlas colored with the available continuous properties. Figure S4: MAP4 TMAP of NPAtlas colored with the ranked continuous properties. Figure S5: MAP4 TMAP of NPAtlas colored with the available categorical properties. Table S1, Figure S6: MAP4 SVMN classification of known non-microbial natural products and their structures.

Author Contributions: A.C. designed and realized the project and wrote the paper. J.-L.R. designed and supervised the project and wrote the paper. All authors have read and agreed to the published version of the manuscript

Funding: This work was supported financially by the Swiss National Science Foundation, Grant no. 200020_178998.

Conflicts of Interest: The authors declare no conflict of interest.

\section{References}

1. Pham, J.V.; Yilma, M.A.; Feliz, A.; Majid, M.T.; Maffetone, N.; Walker, J.R.; Kim, E.; Cho, H.J.; Reynolds, J.M.; Song, M.C.; et al. A Review of the Microbial Production of Bioactive Natural Products and Biologics. Front. Microbiol. 2019, 10, 1404. [CrossRef] [PubMed]

2. Chen, Y.; de Bruyn Kops, C.; Kirchmair, J. Data Resources for the Computer-Guided Discovery of Bioactive Natural Products. J. Chem. Inf. Model. 2017, 57, 2099-2111. [CrossRef] [PubMed]

3. Osada, H.; Nogawa, T. Systematic isolation of microbial metabolites for natural products depository (NPDepo). Pure Appl. Chem. 2011, 84, 1407-1420. [CrossRef]

4. Grabowski, K.; Baringhaus, K.-H.; Schneider, G. Scaffold diversity of natural products: Inspiration for combinatorial library design. Nat. Prod. Rep. 2008, 25, 892-904. [CrossRef] [PubMed]

5. Grisoni, F.; Merk, D.; Consonni, V.; Hiss, J.A.; Tagliabue, S.G.; Todeschini, R.; Schneider, G. Scaffold hopping from natural products to synthetic mimetics by holistic molecular similarity. Commun. Chem. 2018, 1, 1-9. [CrossRef]

6. Fraser, L.-A.; Mulholland, D.A.; Fraser, D.D. Classification of limonoids and protolimonoids using neural networks. Phytochem. Anal. 1997, 8, 301-311. [CrossRef]

7. Martínez-Treviño, S.H.; Uc-Cetina, V.; Fernández-Herrera, M.A.; Merino, G. Prediction of Natural Product Classes Using Machine Learning and 13C NMR Spectroscopic Data. J. Chem. Inf. Model. 2020, 7, 3376-3386. [CrossRef]

8. Rupp, M.; Bauer, M.R.; Wilcken, R.; Lange, A.; Reutlinger, M.; Boeckler, F.M.; Schneider, G. Machine Learning Estimates of Natural Product Conformational Energies. PLoS Comput. Biol. 2014, 10, e1003400. [CrossRef]

9. Chen, Y.; Stork, C.; Hirte, S.; Kirchmair, J. NP-Scout: Machine Learning Approach for the Quantification and Visualization of the Natural Product-Likeness of Small Molecules. Biomolecules 2019, 9, 43. [CrossRef]

10. Rupp, M.; Schroeter, T.; Steri, R.; Zettl, H.; Proschak, E.; Hansen, K.; Rau, O.; Schwarz, O.; Müller-Kuhrt, L.; Schubert-Zsilavecz, M.; et al. From Machine Learning to Natural Product Derivatives that Selectively Activate Transcription Factor PPAR $\gamma$. Chem. Med. Chem. 2010, 5, 191-194. [CrossRef] [PubMed]

11. Awale, M.; Sirockin, F.; Stiefl, N.; Reymond, J.-L. Drug Analogs from Fragment-Based Long Short-Term Memory Generative Neural Networks. J. Chem. Inf. Model. 2019, 59, 1347-1356. [CrossRef] [PubMed]

12. Wang, Y.; Jafari, M.; Tang, Y.; Tang, J. Predicting Meridian in Chinese traditional medicine using machine learning approaches. PLoS Comput. Biol. 2019, 15. [CrossRef] [PubMed]

13. Zhang, R.; Li, X.; Zhang, X.; Qin, H.; Xiao, W. Machine learning approaches for elucidating the biological effects of natural products. Nat. Prod. Rep. 2020. [CrossRef] [PubMed]

14. Van Santen, J.A.; Jacob, G.; Singh, A.L.; Aniebok, V.; Balunas, M.J.; Bunsko, D.; Neto, F.C.; Castaño-Espriu, L.; Chang, C.; Clark, T.N.; et al. The Natural Products Atlas: An Open Access Knowledge Base for Microbial Natural Products Discovery. ACS Cent. Sci. 2019, 5, 1824-1833. [CrossRef] [PubMed]

15. Dice, L.R. Measures of the Amount of Ecologic Association between Species. Ecology 1945, 26, $297-302$. [CrossRef]

16. Rogers, D.; Hahn, M. Extended-Connectivity Fingerprints. J. Chem. Inf. Model. 2010, 50, 742-754. [CrossRef]

17. Capecchi, A.; Probst, D.; Reymond, J.-L. One molecular fingerprint to rule them all: Drugs, biomolecules, and the metabolome. J. Cheminform. 2020, 12, 43. [CrossRef]

18. Carhart, R.E.; Smith, D.H.; Venkataraghavan, R. Atom pairs as molecular features in structure-activity studies: Definition and applications. J. Chem. Inf. Comput. Sci. 1985, 25, 64-73. [CrossRef] 
19. Jin, X.; Awale, M.; Zasso, M.; Kostro, D.; Patiny, L.; Reymond, J.L. PDB-Explorer: A web-based interactive map of the protein data bank in shape space. BMC Bioinform. 2015, 16, 339. [CrossRef]

20. Di Bonaventura, I.; Jin, X.; Visini, R.; Probst, D.; Javor, S.; Gan, B.H.; Michaud, G.; Natalello, A.; Doglia, S.M.; Kohler, T.; et al. Chemical space guided discovery of antimicrobial bridged bicyclic peptides against Pseudomonas aeruginosa and its biofilms. Chem. Sci. 2017, 8, 6784-6798. [CrossRef]

21. Capecchi, A.; Awale, M.; Probst, D.; Reymond, J.-L. PubChem and ChEMBL beyond Lipinski. Mol. Inform. 2019. [CrossRef] [PubMed]

22. Capecchi, A.; Zhang, A.; Reymond, J.-L. Populating Chemical Space with Peptides Using a Genetic Algorithm. J. Chem. Inf. Model. 2020, 60, 121-132. [CrossRef] [PubMed]

23. Probst, D.; Reymond, J.-L. A probabilistic molecular fingerprint for big data settings. J. Cheminform. 2018, 10, 66. [CrossRef] [PubMed]

24. Probst, D.; Reymond, J.-L. Visualization of very large high-dimensional data sets as minimum spanning trees. J. Cheminform. 2020, 12, 12. [CrossRef]

25. Van der Maaten, L.; Hinton, G. Visualizing Data using t-SNE. J. Mach. Learn. Res. 2008, 9, 2579-2605.

26. McInnes, L.; Healy, J.; Melville, J. UMAP: Uniform Manifold Approximation and Projection for Dimension Reduction. arXiv 2018, arXiv:1802.03426.

27. Schneider, N.; Sayle, R.A.; Landrum, G.A. Get Your Atoms in Order-An Open-Source Implementation of a Novel and Robust Molecular Canonicalization Algorithm. J. Chem. Inf. Model. 2015, 55, 2111-2120. [CrossRef]

28. RDKit. Available online: https://www.rdkit.org/ (accessed on 25 September 2018).

29. Dang, Q.H. Secure Hash Standard; National Institute of Standards and Technology: Gaithersburg, MD, USA, 2015.

30. Broder, A.Z.; Charikar, M.; Frieze, A.M.; Mitzenmacher, M. Min-wise Independent Permutations. J. Comput. Syst. Sci. 1998, 60, 327-336. [CrossRef]

31. Bawa, M.; Condie, T.; Ganesan, P. LSH forest: Self-tuning indexes for similarity search. In Proceedings of the 14th international conference on World Wide Web, Chiba, Japan, 10-14 May 2005; Association for Computing Machinery: New York, NY, USA, 2005; pp. 651-660.

32. Kruskal, J.B. On the shortest spanning subtree of a graph and the traveling salesman problem. Proc. Am. Math. Soc. 1956, 7, 48-50. [CrossRef]

33. Probst, D.; Reymond, J.-L.; Wren, J. FUn: A framework for interactive visualizations of large, high-dimensional datasets on the web. Bioinformatics 2018, 34, 1433-1435. [CrossRef]

34. Wildman, S.A.; Crippen, G.M. Prediction of Physicochemical Parameters by Atomic Contributions. J. Chem. Inf. Comput. Sci. 1999, 39, 868-873. [CrossRef]

35. Shi, C.; Borchardt, T.B. JRgui: A Python Program of Joback and Reid Method. ACS Omega 2017, 2, 8682-8688. [CrossRef] [PubMed]

36. Joback, K.G.; Reid, R.C. Estimation of Pure-Component Properties from Group-Contributions. Chem. Eng. Commun. 1987, 57, 233-243. [CrossRef]

37. Lipinski, C.A.; Lombardo, F.; Dominy, B.W.; Feeney, P.J. Experimental and computational approaches to estimate solubility and permeability in drug discovery and development settings. Adv. Drug Deliv. Rev. 1997, 23, 3-25. [CrossRef]

38. Daylight. Available online: https://www.daylight.com/ (accessed on 17 July 2020).

39. Virtanen, P.; Gommers, R.; Oliphant, T.E.; Haberland, M.; Reddy, T.; Cournapeau, D.; Burovski, E.; Peterson, P.; Weckesser, W.; Bright, J.; et al. SciPy 1.0: Fundamental Algorithms for Scientific Computing in Python. Nat. Methods 2020, 17, 261-272. [CrossRef]

40. Noble, W.S. What is a support vector machine? Nat. Biotechnol. 2006, 24, 1565-1567. [CrossRef]

41. Platt, J.C. Probabilistic outputs for support vector machines and comparisons to regularized likelihood methods. In Advances in Large Margin Classifiers; MIT Press: Cambridge, MA, USA, 1999; pp. 61-74.

42. Vert, J.P.; Tsuda, K.; Schölkopf, B. A Primer on Kernel Methods: In Kernel Methods in Computational Biology; Biologische Kybernetik: Cambridge, MA, USA, 2004; pp. 35-70.

43. Pedregosa, F.; Varoquaux, G.; Gramfort, A.; Michel, V.; Thirion, B.; Grisel, O.; Blondel, M.; Prettenhofer, P.; Weiss, R.; Dubourg, V.; et al. Scikit-learn: Machine Learning in Python. J. Mach. Learn. Res. 2011, 12, 2825-2830. 
44. Gallegos, D.A.; Saurí, J.; Cohen, R.D.; Wan, X.; Videau, P.; Vallota-Eastman, A.O.; Shaala, L.A.; Youssef, D.T.A.; Williamson, R.T.; Martin, G.E.; et al. Jizanpeptins, Cyanobacterial Protease Inhibitors from a Symploca sp. Cyanobacterium Collected in the Red Sea. J. Nat. Prod. 2018, 81, 1417-1425. [CrossRef]

45. Mao, X.-M.; Xu, W.; Li, D.; Yin, W.-B.; Chooi, Y.-H.; Li, Y.-Q.; Tang, Y.; Hu, Y. Epigenetic Genome Mining of an Endophytic Fungus Leads to the Pleiotropic Biosynthesis of Natural Products. Angew. Chem. Int. Ed. 2015, 54, 7592-7596. [CrossRef]

46. Dion, H.W.; Woo, P.W.K.; Willmer, N.E.; Kern, D.L.; Onaga, J.; Fusari, S.A. Butirosin, a New Aminoglycosidic Antibiotic Complex: Isolation and Characterization. Antimicrob. Agents Chemother. 1972, 2, 84-88. [CrossRef]

47. Tatsuda, D.; Momose, I.; Someno, T.; Sawa, R.; Kubota, Y.; Iijima, M.; Kunisada, T.; Watanabe, T.; Shibasaki, M.; Nomoto, A. Quinofuracins A-E, Produced by the Fungus Staphylotrichum boninense PF1444, Show p53-Dependent Growth Suppression. J. Nat. Prod. 2015, 78, 188-195. [CrossRef]

48. Zhang, Y.; Liu, S.; Liu, H.; Liu, X.; Che, Y. Cycloaspeptides F and G, Cyclic Pentapeptides from a Cordyceps-Colonizing Isolate of Isaria farinosa. J. Nat. Prod. 2009, 72, 1364-1367. [CrossRef] [PubMed]

49. Tsuji, N.; Kobayashi, M.; Kamigauchi, T.; Yoshimura, Y.; Terui, Y. New glycopeptide antibiotics. I. The structures of orienticins. J. Antibiot. 1988, 41, 819-822. [CrossRef]

50. Kim, M.C.; Hwang, E.; Kim, T.; Ham, J.; Kim, S.Y.; Kwon, H.C. Nocatriones A and B, Photoprotective Tetracenediones from a Marine-Derived Nocardiopsis sp. J. Nat. Prod. 2014, 77, 2326-2330. [CrossRef] [PubMed]

51. Li, X.-B.; Zhou, Y.-H.; Zhu, R.-X.; Chang, W.-Q.; Yuan, H.-Q.; Gao, W.; Zhang, L.-L.; Zhao, Z.-T.; Lou, H.-X. Identification and Biological Evaluation of Secondary Metabolites from the Endolichenic Fungus Aspergillus versicolor. Chem. Biodivers. 2015, 12, 575-592. [CrossRef] [PubMed]

52. Spyere, A.; Rowley, D.C.; Jensen, P.R.; Fenical, W. New Neoverrucosane Diterpenoids Produced by the Marine Gliding Bacterium Saprospira grandis. J. Nat. Prod. 2003, 66, 818-822. [CrossRef]

53. Yamamoto, T.; Izumi, N.; Ui, H.; Sueki, A.; Masuma, R.; Nonaka, K.; Hirose, T.; Sunazuka, T.; Nagai, T.; Yamada, H.; et al. Wickerols A and B: Novel anti-influenza virus diterpenes produced by Trichoderma atroviride FKI-3849. Tetrahedron 2012, 68, 9267-9271. [CrossRef]

54. Mitchell, J.B.O. Machine learning methods in chemoinformatics. Wiley Interdiscip. Rev. Comput. Mol. Sci. 2014, 4, 468-481. [CrossRef]

55. Lanzoni, O.; Sabaneyeva, E.; Modeo, L.; Castelli, M.; Lebedeva, N.; Verni, F.; Schrallhammer, M.; Potekhin, A.; Petroni, G. Diversity and environmental distribution of the cosmopolitan endosymbiont "Candidatus Megaira". Sci. Rep. 2019, 9, 1179. [CrossRef]

56. Zhu, G.; Hou, C.; Yuan, W.; Wang, Z.; Zhang, J.; Jiang, L.; Karthik, L.; Li, B.; Ren, B.; Lv, K.; et al. Molecular networking assisted discovery and biosynthesis elucidation of the antimicrobial spiroketals epicospirocins. Chem. Commun. 2020. [CrossRef]

57. Cheng, X.; Liang, X.; Zheng, Z.-H.; Zhang, X.-X.; Lu, X.-H.; Yao, F.-H.; Qi, S.-H. Penicimeroterpenoids A-C, Meroterpenoids with Rearrangement Skeletons from the Marine-Derived Fungus Penicillium sp. SCSIO 41512. Org. Lett. 2020. [CrossRef] [PubMed]

58. Kwon, Y.; Shin, J.; Nam, K.; An, J.S.; Yang, S.-H.; Hong, S.-H.; Bae, M.; Moon, K.; Cho, Y.; Woo, J.; et al. Rhizolutin, a novel 7/10/6-tricyclic dilactone, dissociates misfolded protein aggregates and reduces apoptosis/inflammation associated with Alzheimer's disease. Angew. Chem. Int. Ed. 2020. [CrossRef]

59. Xu, Z.F.; Bo, S.T.; Wang, M.J.; Shi, J.; Jiao, R.H.; Sun, Y.; Xu, Q.; Tan, R.; Ge, H.M. Discovery and biosynthesis of bosamycin from Streptomyces sp. 120454. Chem. Sci. 2020. [CrossRef]

60. Luyen, N.D.; Huong, L.M.; Thi Hong Ha, T.; Cuong, L.H.; Thi Hai Yen, D.; Nhiem, N.X.; Tai, B.H.; Gardes, A.; Kopprio, G.; Van Kiem, P. Aspermicrones A-C, novel dibenzospiroketals from the seaweed-derived endophytic fungus Aspergillus micronesiensis. J. Antibiot. 2019, 72, 843-847. [CrossRef]

61. Kosemura, S. Meroterpenoids from Penicillium citreo-viride B. IFO 4692 and 6200 hybrid. Tetrahedron 2003, 59, 5055-5072. [CrossRef]

62. Endo, A. Monacolin K, a new hypocholesterolemic agent that specifically inhibits 3-hydroxy-3-methylglutaryl coenzyme A reductase. J. Antibiot. 1980, 33, 334-336. [CrossRef]

63. Ji, G.; Beavis, R.; Novick, R.P. Bacterial Interference Caused by Autoinducing Peptide Variants. Science 1997, 276, 2027-2030. [CrossRef] 
64. Wu, Y.; Liao, H.; Liu, L.-Y.; Sun, F.; Chen, H.-F.; Jiao, W.-H.; Zhu, H.-R.; Yang, F.; Huang, G.; Zeng, D.-Q.; et al. Phakefustatins A-C: Kynurenine-Bearing Cycloheptapeptides as RXR $\alpha$ Modulators from the Marine Sponge Phakellia fusca. Org. Lett. 2020. [CrossRef]

65. Naman, C.B.; Rattan, R.; Nikoulina, S.E.; Lee, J.; Miller, B.W.; Moss, N.A.; Armstrong, L.; Boudreau, P.D.; Debonsi, H.M.; Valeriote, F.A.; et al. Integrating Molecular Networking and Biological Assays To Target the Isolation of a Cytotoxic Cyclic Octapeptide, Samoamide A, from an American Samoan Marine Cyanobacterium. J. Nat. Prod. 2017, 80, 625-633. [CrossRef]

66. Brinkmann, C.M.; Marker, A.; Kurtböke, D.İ. An Overview on Marine Sponge-Symbiotic Bacteria as Unexhausted Sources for Natural Product Discovery. Diversity 2017, 9, 40. [CrossRef]

67. Han, M.; Liu, F.; Zhang, F.; Li, Z.; Lin, H. Bacterial and archaeal symbionts in the South China Sea sponge Phakellia fusca: Community structure, relative abundance, and ammonia-oxidizing populations. Mar. Biotechnol. 2012, 14, 701-713. [CrossRef] [PubMed]

68. Sorokina, M.; Steinbeck, C. Review on natural products databases: Where to find data in 2020. J. Cheminform. 2020, 12, 20. [CrossRef]

69. Chen, Y.; Kirchmair, J. Cheminformatics in Natural Product-Based Drug Discovery. Mol. Inform. 2020. [CrossRef] [PubMed]

(C) 2020 by the authors. Licensee MDPI, Basel, Switzerland. This article is an open access article distributed under the terms and conditions of the Creative Commons Attribution (CC BY) license (http://creativecommons.org/licenses/by/4.0/). 